\title{
Conjectural Contriving of Orthogonal Frequency Division Multiplexing (OFDM) Transreceiver with the Aid of Phase Shift Keying (PSK) and a 256 bit Grayscale Image
}

\author{
Sudipta Ghosh \\ Student, SECE \\ Lovely Professional University \\ Jalandhar, INDIA
}

\author{
Chandika Mohan Babu \\ Asst. Professor, SECE \\ Lovely Professional University \\ Jalandhar, INDIA
}

\begin{abstract}
Orthogonal Frequency Division Multiplexing (OFDM) is one of the latest modulation schemes that is being used for high speed data transmission. OFDM is basically based on the principle of orthogonality, wherein the sub carriers are kept orthogonal. In order to decide the number of symbols per carrier we can use QAM and PSK. In this work of ours we have used PSK, specifically BPSK (i.e. 2 symbols per carrier). The basic purpose of this paper is to provide a MATLAB simulation of the basic processing involved in the generation and reception of an OFDM signal using a 256 bit gray scale image in a physical channel and to provide a description of each of the steps involved. In this paper we have two fields i.e. image processing and communication.
\end{abstract}

\section{General Terms}

Fast Fourier Transform (FFT), Inverse Fourier Transform (IFFT), Pulse Shaping, Filters, Phase Shift Keying (PSK)

\section{Keywords}

Orthogonal Frequency Division Multiplexing (OFDM), Gray scale image

\section{INTRODUCTION}

In telecommunication, a communication system is a collection of individual communication networks, transmission systems, relay stations, tributary stations, and data terminal equipment usually capable of interconnection and inter operation to form an integrated whole. The components of a communication system serve a common purpose, are technically compatible, use common procedures, respond to controls, and operate in unison. Telecommunications is a method of communication (e.g. for sports broadcasting, mass, media, etc.). A communication sub system is a functional unit or operational assembly that is smaller than the larger assembly under consideration. The principle of multi-carrier transmission is to convert a serial high rate data stream on to multiple parallel low rate sub-streams. Each sub-stream is modulated on another sub-carrier. Since the symbol rate on each subcarrier is much less than the initial serial data symbol rate, the effects of delay spread, i.e. ISI, significantly decrease, reducing the complexity of the equalizer. OFDM is a low complex technique used to modulate multiple sub-carriers efficiently by using digital signal processing [9]. Note that the three-dimensional time/frequency/power density representation is used to illustrate the principle of various multi-carrier and multi-carrier spread spectrum systems. A cuboid indicates the three-dimensional time/frequency/power density range of the signal, in which most of the signal energy is located and does not make any statement about the pulse or spectrum shaping. As such it has received much attention and has been proposed for many other applications, including local area networks and personal communication systems. OFDM is a type of multichannel modulation that divides a given channel into many parallel sub channels or subcarriers, so that multiple symbols are sent in parallel. The type of OFDM that we will describe in this article uses the discrete Fourier transform (DFT) with a cyclic prefix. DFT (implemented with a fast Fourier transform (FFT)) and the cyclic prefix have made OFDM both practical and attractive to the radio link designer. A similar multichannel modulation scheme, discrete multitone (DMT) modulation, has been developed for static channels such as the digital subscriber loop. DMT also uses DFTs and the cyclic prefix but has the additional feature of bit-loading which is generally not used in OFDM.

OFDM also has some drawbacks. Because OFDM divides a given spectral allotment into many narrow subcarriers each with inherently small carrier spacing, it is sensitive to carrier frequency errors. Furthermore, to preserve the orthogonality between subcarriers, the amplifiers need to be linear. OFDM systems also have a high peak-to-average power ratio or crest-factor, which may require a large amplifier power backoff and a large number of bits in the analog-to-digital (A/D) and digital-to-analog (D/A) designs. All these requirements can put a high demand on the transmitter and receiver design [10].

\section{ORTHOGONAL FREQUENCY DIVISION MULTIPLEXING (OFDM)}

OFDM is a multi-carrier modulation technique where data symbols modulate a sub-carrier which is taken from orthogonally separated sub-carriers with a separation of ' $\mathrm{fk}$ ' within each sub-carrier. Here, the spectra of sub-carrier are overlapping; but the sub-carrier signals are mutually orthogonal, which is utilizing the bandwidth very efficiently. To maintain the orthogonality, the minimum separation between the sub-carriers should be ' $f_{\mathrm{K}}$ ' to avoid ICI (Inter Carrier Interference).By choosing the sub-carrier spacing properly in relation to the channel coherence bandwidth. OFDM can be used to convert a frequency selective channel into a parallel collection of frequency flat sub-channels. 
Techniques that are appropriate for flat fading channels can then be applied in a straight forward fashion [4].

\section{SIGNAL MODEL}

A communication system with multi-carrier modulation transmits $\mathrm{N}_{\mathrm{C}}$ complex-valued source symbol $\mathrm{S}_{\mathrm{N}}, \mathrm{N}=0$, $\ldots, \mathrm{N}_{\mathrm{C}}-1$, in parallel on $\mathrm{N}_{\mathrm{C}}$ sub-carriers. The source symbols may, for instance, be obtained after source and channel coding, interleaving, and symbol mapping [2]. The source symbol duration $T_{S}$ of the serial data symbols results after serial- to-parallel conversion in the OFDM symbol duration.

$$
T_{s}=N_{c} T_{d}
$$

The principle of OFDM is to modulate the $\mathrm{N}_{\mathrm{C}}$ sub-streams on sub-carriers with a spacing of

$$
F_{s}=1 / T_{s}
$$

in order to achieve orthogonality between the signals on the $\mathrm{Nc}$ sub-carriers, presuming a rectangular pulse shaping. The $\mathrm{Nc}$ parallel modulated source symbols $\mathrm{Sn}, \mathrm{n}=0, \ldots, \mathrm{N}_{\mathrm{C}}-1$, are referred to as an OFDM symbol [8]. The complex envelope of an OFDM symbol with rectangular pulse shaping has the form

$$
x(t)=\frac{1}{N_{c}} \sum_{n=0}^{N_{c}-1} S_{n} e^{j 2 \pi f_{n} t} \quad, 0 \leq t<T_{s}
$$

The Nc sub-carrier frequencies are located at

$$
f_{n}=\frac{n}{T_{s}}, n=0, \ldots \ldots, N_{c}-1
$$

The symbols $S_{n}, n=0, \ldots, N_{C}-1$, are transmitted with equal power. The dotted curve illustrates the power density spectrum of the first modulated sub-carrier and indicates the construction of the overall power density spectrum as the sum of $\mathrm{N}_{\mathrm{C}}$ individual power density spectra, each shifted by $\mathrm{F}_{\mathrm{S}}$. For large values of $\mathrm{N}_{\mathrm{C}}$, the power density spectrum becomes flatter in the normalized frequency range of $-0.5{ }_{-} \mathrm{fTd} \mathrm{d}_{-} 0.5$ containing the $\mathrm{N}_{\mathrm{C}}$ subchannels. Only sub-channels near the band edges contribute to the out-of-band power emission. Therefore, as $\mathrm{N}_{\mathrm{C}}$ becomes large, the power density spectrum approaches that of single carrier modulation with ideal Nyquist filtering [1]. A key advantage of using OFDM is that multi-carrier modulation can be implemented in the discrete domain by using an IDFT, or a more computationally efficient IFFT. When sampling the complex envelope $x(t)$ of an OFDM symbol with rate $1 / \mathrm{Td}$ the samples are

$$
x_{v}=\frac{1}{N_{c}} \sum_{0}^{N_{c-1}} S_{n} e^{j 2 \pi n v / N_{c}}, v=0, \ldots \ldots, N_{c}-1
$$

The normalized power spectrum of OFDM is shown in Fig. 1

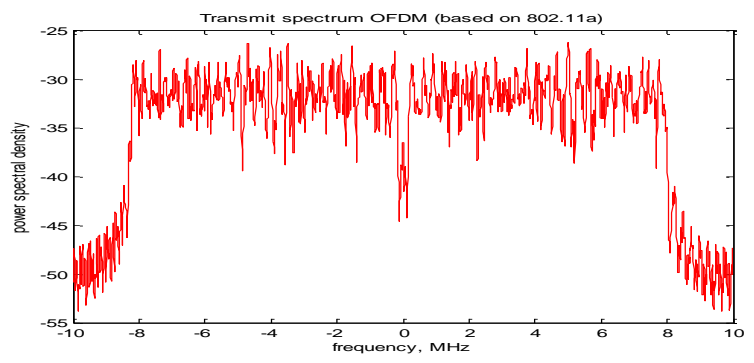

Figure 1. Normalized power spectrum of an OFDM signal

\section{Phase Shift Keying (PSK)}

Phase-shift keying (PSK) is a digital modulation scheme that conveys data by changing, or modulating, the phase of a reference signal (the carrier wave). Any digital modulation scheme uses a finite number of distinct signals to represent digital data. PSK uses a finite number of phases; each assigned a unique pattern of binary digits. Usually, each phase encodes an equal number of bits. Each pattern of bits forms the symbol that is represented by the particular phase. The demodulator, which is designed specifically for the symbol-set used by the modulator, determines the phase of the received signal and maps it back to the symbol it represents, thus recovering the original data. This requires the receiver to be able to compare the phase of the received signal to a reference signal such a system is termed coherent (and referred to as CPSK). Alternatively, instead of operating with respect to a constant reference wave, the broadcast can operate with respect to itself. Changes in phase of a single broadcast waveform can be considered the significant items. In this system, the demodulator determines the changes in the phase of the received signal rather than the phase (relative to a reference wave) itself. Since this scheme depends on the difference between successive phases, it is termed differential phase-shift keying (DPSK). DPSK can be significantly simpler to implement than ordinary PSK since there is no need for the demodulator to have a copy of the reference signal to determine the exact phase of the received signal (it is a non-coherent scheme). In exchange, it produces more erroneous demodulations [1].

The simplest form of PSK is binary phase-shift keying (BPSK), where $\mathrm{N}=1$ and $\mathrm{M}=2$. Therefore, with BPSK, two phases $\left(2^{1}=2\right)$ are possible for the carrier. One phase represents logic 1 , and the other phase represents logic 0 . As the input digital signal changes state (i.e., from 1 to 0 or from a 0 to a 1), the phase of the output carrier shifts between two angles that are separated by $180^{\circ}$. Hence, other names for BPSK are phase reversal keying (PRK) and bi phase modulation. BPSK is a form of square-wave modulation of a continuous wave (CW) signal [3]. A binary phaseshift keying (BPSK) signal can be defined by

$$
s(t)=A m(t) \cos \left(2 \pi f_{c} t\right), 0 \leq t \leq T
$$

where $\mathrm{A}$ is a constant, $\mathrm{m}(\mathrm{t})=+1$ or $-1, \mathrm{fc}$ is the carrier frequency, and $\mathrm{T}$ is the bit duration. The signal has a power $\mathrm{P}$ $=A 2 / 2$, so that $A=2 P$. Thus equation 6 can be written as

$$
s(t)= \pm \sqrt{2 P} \cos \left(2 \pi f_{c} t\right)
$$




$$
\begin{aligned}
& = \pm \sqrt{P T} \sqrt{\frac{2}{T}} \cos \left(2 \pi f_{c} t\right) \\
& = \pm \sqrt{E} \sqrt{\frac{2}{T}} \cos \left(2 \pi f_{c} t\right)
\end{aligned}
$$

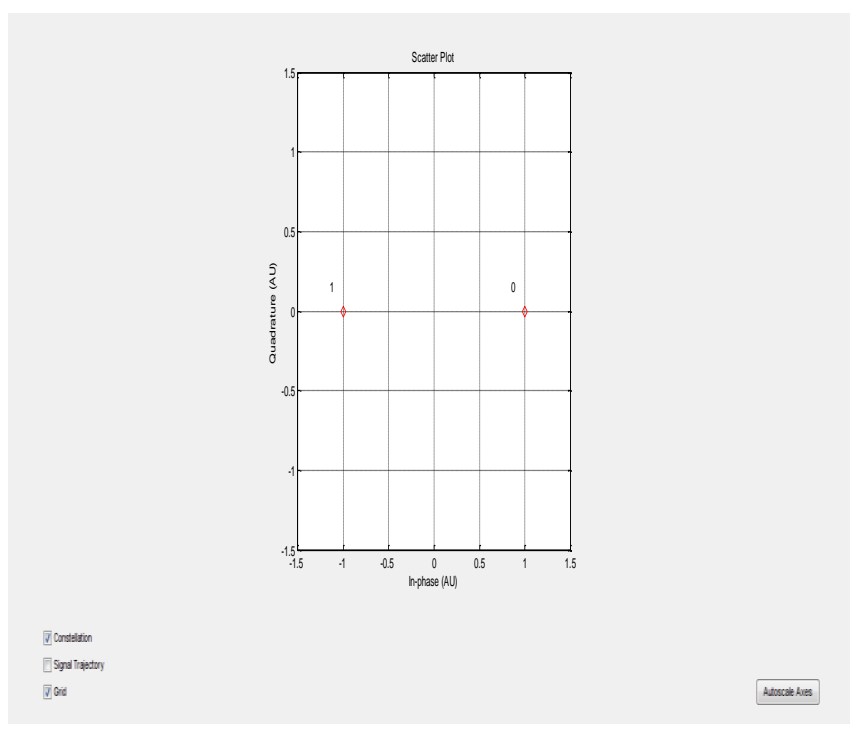

Figure 2. Constellation diagram of BPSK

Figure 3 shows the BPSK signal sequence generated by the binary sequence, the modulating signal and the modulated BPSK signal for the input sequence 00011011

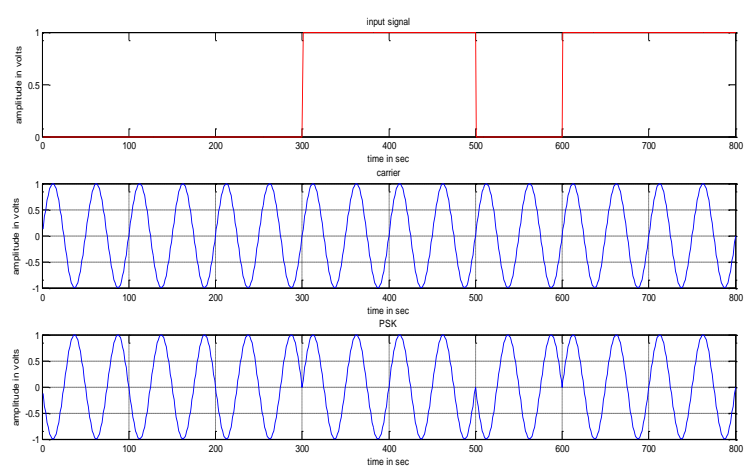

Figure 3. BPSK modulated signal

\section{Transmitter Model using MATLAB}

The core of the OFDM transmitter is the modulator, which modulates the input data stream frame by frame. Data is divided into frames based on the variable symb_per_frame, which refers to the number of symbols per frame per carrier. It is defined by: symb_per_frame $=\operatorname{ceil}\left(2^{\wedge} 13\right.$ /carrier_count $)$. This limits the total number of symbols per frame (symb_per_frame $*$ carrier_count) within the interval of $\left[2^{\wedge} 13,2^{*}\left(2^{\wedge} 13-1\right)\right]$, or $[8192,16382]$. However, the number of carriers typically would not be much greater than 1000 in this simulation, thus the total number of symbols per frame would typically be under 10,000 . This is an experimentally reasonable number of symbols that one frame should keep under for this MATLAB program to run efficiently; thereby symbol per frameis defined by the equation shown above. If the total number of symbols in a data stream to be transmitted is less than the total number of symbols per frame, the data would not be divided into frames and would be modulated all at once. Even if the data stream is not sufficiently long enough to be divided into multiple frames, two frame guards with all zero values and in a length of one symbol period are still added to both ends of the modulated time signal.

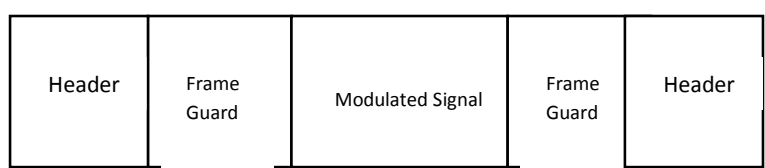

Figure 5. Frame diagram of modulated signal

This is to assist the receiver to locate the beginning of the substantial portion of the time signal. For modulated signals with multiple frames, a frame guard is inserted in between any two adjacent frames as well as both ends of the cascaded time signal. Finally, a pair of headers is padded to both ends of the guarded series of frames. The headers are scaled to the RMS level of the modulated time signal. Figure 4 shows the 256 bit gray scale input image. Using the above methodologies and formulas a transmitter system was developed in MATLAB. The simulation plots obtained are shown in figures 5 to 8 .

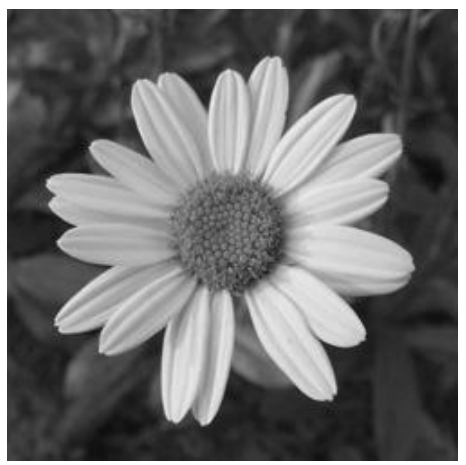

Figure 5. Input 256 bit grayscale image

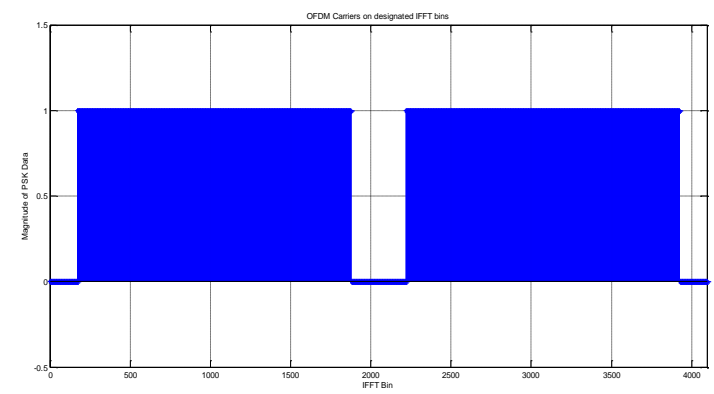

Figure 6. OFDM carriers

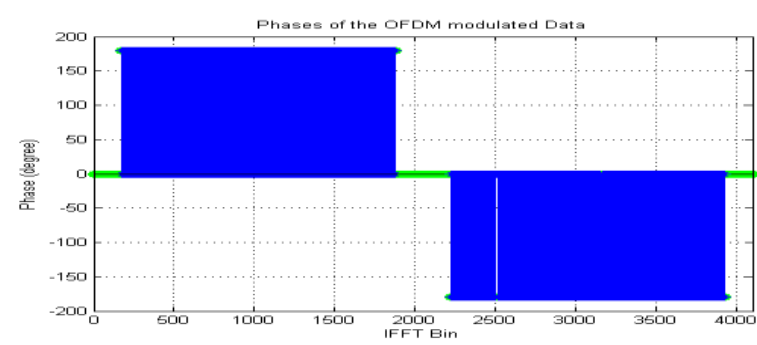

Figure 7. Phase of the OFDM modulated data 


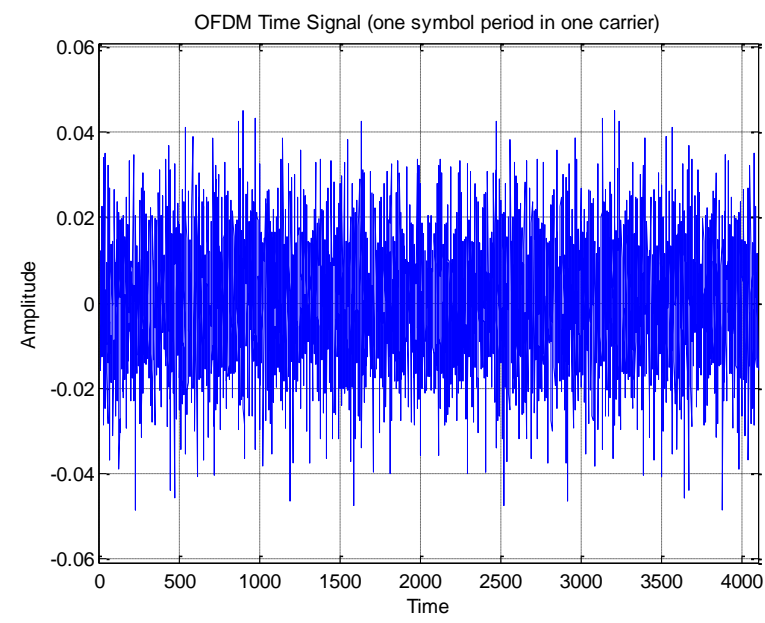

Figure 8. OFDM time signal

We have used AWGN noise in order to simulate a noisy channel interface. Figure 10 shows a screen shot of the data that was used as input and the various data obtained during the simulation. In this simulation we used the length of the IIFT as 4096 and also used 1705 sub carriers which is very much similar to that used in DVB-T [9], another application of OFDM.

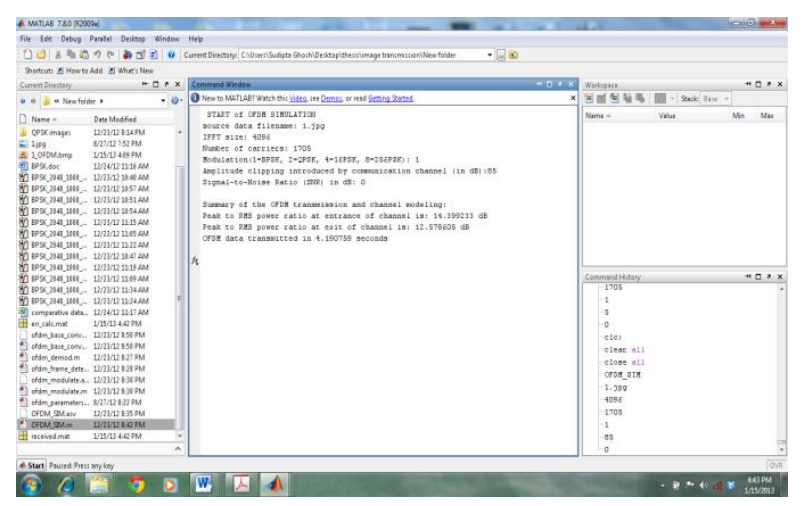

Figure 9. Screenshot of the simulated data

\section{RECEIVER}

There are various ways of frame detection in an OFDM modulated system. Some of the methods that are commonly pressed into action are detection of frame, null power detection and power correlation factors. Here we have used the frame detection method. Once the signal is received, it is selectively processed length wise in the frame detector. The purpose of doing this is to find out the start of the frame. Only the first frame's portion is taken relatively larger in order to take the header frame into account. The selected portion of received signal is sampled into a shorter discrete signal with a sampling rate defined by the system. A moving sum is taken over this sampled signal. The index of the minimum of the sampled signal is approximately the start of the frame guard while one symbol period further from this index is the approximate location for the start of the useful signal frame. The frame detector will then collect a moving sum of the input signal from about $10 \%$ of one symbol period earlier than the approximate start of the frame guard to about one third of symbol period further than the approximate start of the useful signal frame. The first portion, with a length of one less than a symbol period of this moving sum is discarded. The first minimum of this moving sum is the detected start of the useful signal frame. The received OFDM signal is typically demodulated frame by frame. The OFDM receiver shows the progress of frames being demodulated.

However, the total number of frames may vary by a wide range depending on the total amount of information transmitted via the OFDM system. In order to prevent the simulation screen from being overwhelmed by error messages, a specific condition is implemented. The condition is $\operatorname{rem}(\mathrm{k}, \max ($ floor(num_frame/10),1))==0 where $\mathrm{k}$ is the variable to indicate the $\mathrm{k}$-th frame being modulated, and num_frame is the total number of frames. It means that for a total number of frames being 20 or more, it only displays the $\mathrm{n}$-th frame when $\mathrm{n}$ is an integer multiple of the round-down integer of a tenth of the total number of frames; and for a total number of frames being 19 or less, it shows every frame that is being modulated. This would keep the total number of displays within the range from 11 to 19 , provided that the total number of frames is more than 10; otherwise, it simply shows as many messages as the total number of frames.

Figures 10 to 11 shows the magnitude and phase of the received demodulated frame.

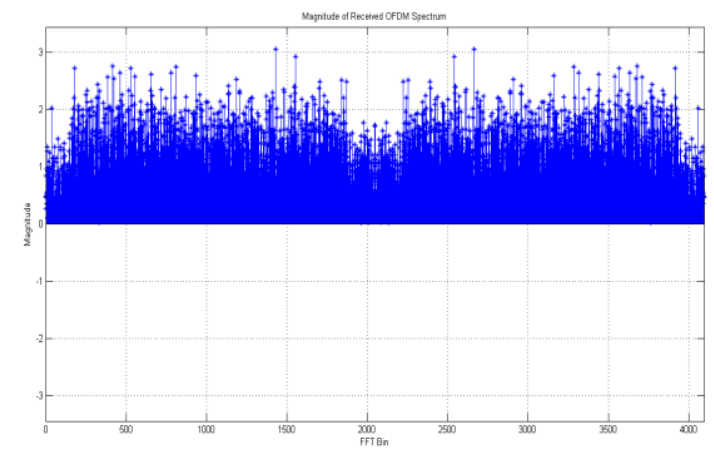

Figure 10. Magnitude of the received OFDM signal

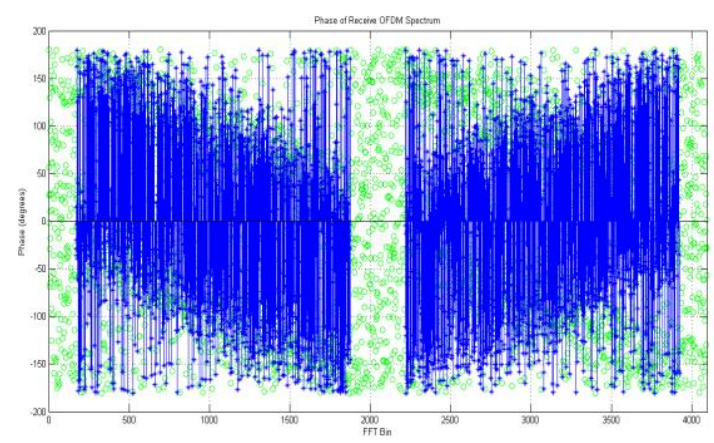

Figure 11. Phase of the received OFDM signal

Since in this system we have used an image as an input we need to see the effect of varying signal to noise ratio (SNR) on the input image. The communication channel is using Additive White Gaussian Noise (AWGN) as the noise model. Figures 12 to 13 shows the effect of varying SNR on the input image for IFFT size 4096, 1705 carriers, and BPSK . 


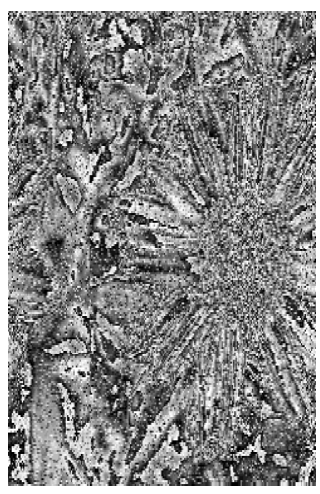

Figure 12. Received image with $\mathrm{SNR}=0$

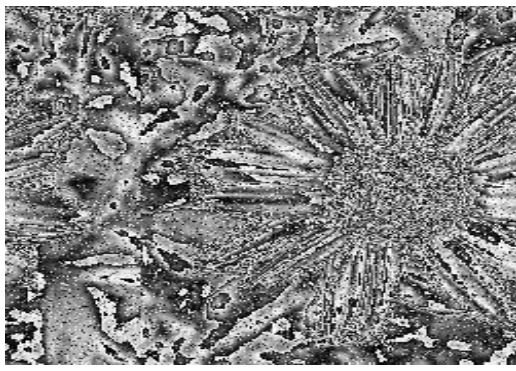

Figure 13. Received image with $S N R=1$

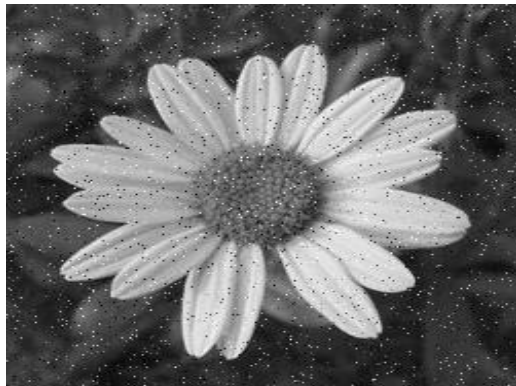

Figure 14. Received image with $S N R=4$

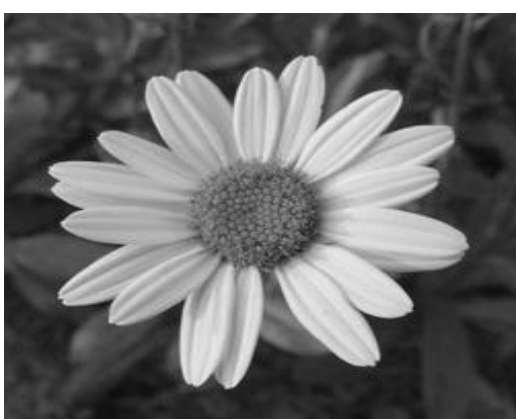

Figure 15. Received image with $\mathrm{SNR}=10$

Figure 16 to 17 shows the graphical representation of the data obtained in the form of BER, SNR, and percentage pixel error. The graph shows us a comparison of the data obtained. The data obtained was a part of the program coding that was designed in MATLAB.

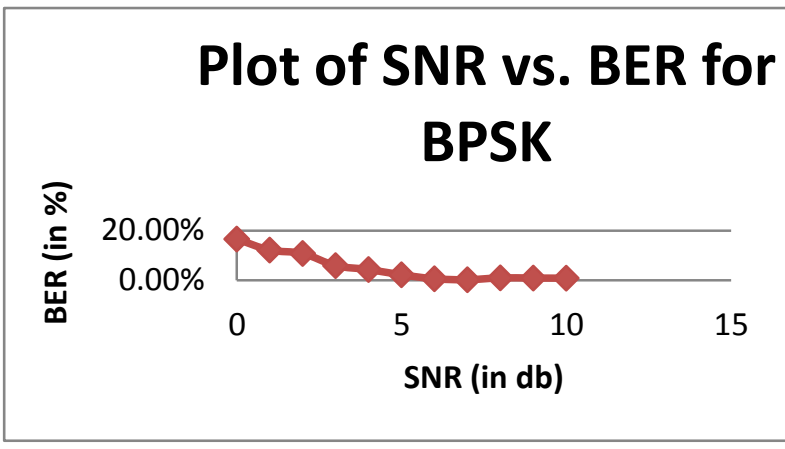

Figure 16. Plot of SNR vs. BPSK

\section{Plot of SNR vs. Pixel Error for BPSK}

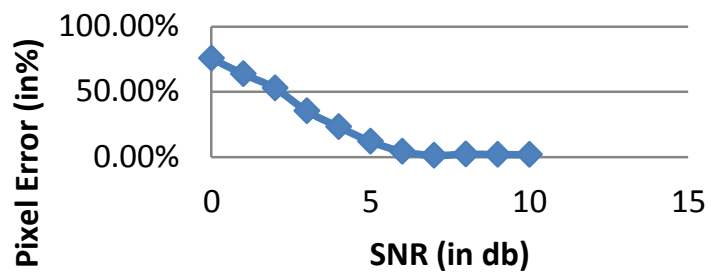

Figure 17. Plot of SNR vs. percentage pixel error

Table 1 gives us the tabular data that was calculated in the coding and gives us a clear numerical value of the data obtained in the form of BER, SNR, and percentage pixel error.

\begin{tabular}{|c|c|c|}
\hline \multicolumn{3}{|c|}{ BPSK } \\
\hline SNR & BER & \%pixel error \\
\hline $\mathbf{0}$ & $16.54 \%$ & $75.65 \%$ \\
\hline $\mathbf{1}$ & $12.10 \%$ & $63.70 \%$ \\
\hline $\mathbf{2}$ & $10.73 \%$ & $52.80 \%$ \\
\hline $\mathbf{3}$ & $5.82 \%$ & $35.28 \%$ \\
\hline $\mathbf{4}$ & $4.26 \%$ & $23.12 \%$ \\
\hline $\mathbf{5}$ & $2.14 \%$ & $11.96 \%$ \\
\hline $\mathbf{6}$ & $0.53 \%$ & $4.18 \%$ \\
\hline $\mathbf{7}$ & $0.15 \%$ & $1.16 \%$ \\
\hline $\mathbf{8}$ & $0.89 \%$ & $2.12 \%$ \\
\hline $\mathbf{9}$ & $0.81 \%$ & $1.82 \%$ \\
\hline $\mathbf{1 0}$ & $0.77 \%$ & $1.78 \%$ \\
\hline
\end{tabular}

Table 1. Numerical values of BER and percentage pixel error obtained for a varying range of SNR

\section{CONCLUSION}

In this paper the transmitter and receiver model have been discussed in details along with their spectral plots. We have also shown the effects of varying the SNR while keeping the 
length of IFFT and number of carriers same. It can be clearly seen that as the value of SNR increases the value of BER and percentage pixel error decreases. As a result of this the image received at a higher value of SNR is more clearer than that received at a lower value. This whole paper is based on the simulation results obtained from the simulation of the discussed model in MATLAB. Further studies are being done in order to enhance the system by implementing corrective measures like FEC coding and so on and also to graphically compare the SNR, BER, percentage pixel error for varying ranges of BPSK, QPSK.

\section{ACKNOWLEDGEMENT}

We would like to thank Mr. Chandika Mohan Babu, my guide, and Mr. Ankit Bass for being a constant source of inspiration and support while writing this research paper. Without their help this would not have been a success.

\section{REFERENCES}

[1] David M Beams, "Electrical Measurement, Signal Processing, and Displays", CRC Press.

[2] C. Yuen, Y. Wu, and S. Sun, "Comparative study of open-loop transmit diversity schemes for four transmit antennas in coded OFDM systems," In Proceeding of IEEE conference on Vehicular Technology, pp. 482-485, (Baltimore, MD)September 2004

[3] A. Boariu and D.M. Ionescu "A class of nonorthogonal rate-one space time block codes with controlled interference," IEEE Transactions on Wireless
Communications, Vol. 2, Issue 2, pp. 270-395, March 2003

[4] V. Tarokh, H. Jafarkhani and A. R. Calderbank, "Spacetime block codes from orthogonal designs", IEEE Transactions on Information Theory, Vol. 45, pp. 14561467, July 1999

[5] J. Kim and I. Lee, "Space-time coded OFDM systems with four transmit antennas," In Proceeding of IEEE conference on Vehicular Technology, vol. 2, pp. 2434 2438, September 2004

[6] Orthogonal frequency division multiplexing for highspeed optical transmission, by Ivan B. Djordjevic et al

[7] A Novel Construction Technique For Designing Of Video Application Using Wirless 4G, by M.Suman et al.

[8] Engels M. (ed.), "Wireless OFDM Systems: How to Make Them Work" , Boston: Kluwer Academic Publishers.

[9] OFDM Simulation Using Matlab, http://www.ece.gatech.edu/research/labs/sarl/tutorials/OF DM/Tutorial_web.pdf.

[10] Sudipta Ghosh, Ankit Bass, "Implementation of digital video broadcasting terrestrial (DVB-T) using orthogonal frequency division multiplexing (OFDM) on physical media dependent sub layer", IJCA (International Journal of Computer Application), Volume 44, number 22,April 2012, Pg 20 to 25 